\title{
Covering Games: Approximation through Non-Cooperation *
}

\author{
Martin Gairing \\ Department of Computer Science, University of Liverpool, U.K. \\ m.gairing@liverpool.ac.uk
}

\begin{abstract}
We propose approximation algorithms under game-theoretic considerations. We indroduce and study the general covering problem which is a natural generalization of the well-studied max-n-cover problem. In the general covering problem, we are given a universal set of weighted elements $E$ and $n$ collections of subsets of the elements. The task is to choose one subset from each collection such that the total weight of their union is as large as possible. In our game-theoretic setting, the choice in each collection is made by an independent player. For covering an element, the players receive a payoff defined by a nonincreasing utility sharing function. This function defines the fraction that each covering player receives from the weight of the elements.

We show how to construct a utility sharing function such that every Nash Equilibrium approximates the optimal solution by a factor of $1-\frac{1}{e}$. We also prove that any sequence of unilateral improving steps is polynomially bounded. This gives rise to a polynomial-time local search approximation algorithm whose approximation ratio is best possible.
\end{abstract}

\section{Introduction}

Motivation and Framework. Large scale distributed systems, like the Internet, usually lack a centralized control authority. Instead, they are operated and controlled in a distributed fashion by competing entities - modeled as players which make their decisions in order to optimize their own private utility. Such systems are assumed to end up in a Nash equilibrium [21] - a state in which no player wishes to unilaterally leave her own strategy in order to improve the value of her private utility. However, Nash equilibria are often suboptimal solutions with respect to the social objective function. The price of anarchy [19] is a measure for the performance degradation. It is defined as the worst-case ratio between the values of a social objective function in a Nash equilibrium and in an optimum solution.

As the designer of a distributed system we are faced with the main challenge of how to design the distributed system in order to optimize this social objective function even in the presence of myopic players. However, even if all players

* This work was supported by a fellowship within the Postdoc-Programme of the German Academic Exchange Service (DAAD). 
adhere to some centralized authority, finding the optimum solution with respect to the social objective might be an $\mathcal{N} \mathcal{P}$-hard optimization problem. Approximation algorithms [25] are a powerful tool for coping with intractable optimization problems. In general, they compute (in polynomial time) suboptimal solutions but with a provable performance guarantee. However, approximation algorithms usually presume a centralized authority. In this paper we propose to consider approximation algorithms that take the selfish user behavior into account. More precisely, we propose to design the distributed system in a way that the price of anarchy is optimized and the system is guaranteed to converge to a Nash equilibrium in polynomial time.

For our study of such approximation algorithms we consider a very general covering problem. In covering problems a finite set of elements has to be covered with subsets of the elements. Such problems arise in many contexts: Covering problems can be used to model service installation problems in distributed systems. Moreover, many packing problems and fixed parameter optimization problems can be modeled as a covering problem (see [14]). A well-studied representative is the max-n-cover problem (see e.g. [10,15]): Given a finite set of weighted elements $E$, choose $n$ subsets of the elements (from a given collection of subsets) such that the total weight of their union is as large as possible. We consider a generalization of the max- $n$-cover problem that we call general covering problem. Here, we are given not 1 but $n$ collections of subsets and we have to choose one subset from each collection. Although this generalization seems natural, we are not aware of any previous work on it.

We study the general covering problem as a covering game, where the choice for the subset in each of the $n$ collections is made by an independent player. Covering games are a subclass of the congestion games introduced by Rosenthal [22]. For covering an element, the players receive a payoff defined by a utility sharing function. This function defines the fraction that each covering player receives from the weight of the element and depends only on local parameters. Those parameters are the number of players covering an element and the cardinality of an element (i.e. the number of players that have this element in at least one of their strategies). We only make two natural assumptions on the utility sharing function: First, we assume that it is non-increasing in the number of players covering the element. And second, we want that the payoff to the players for covering an element does not exceed the weight of the element.

The focus of this paper is to design utility sharing functions, such that:

1. In any Nash equilibrium the total weight of the covered elements is as large as possible. Or more precisely, the price of anarchy is maximized.

2. A Nash equilibrium is reached in polynomial time.

Obviously, each utility sharing function that fulfills both of this properties gives rise to a local search approximation algorithm. In fact, we will show that this approach yields essentially the best possible approximation ratio.

Contribution. In this paper, we introduce and study covering games. Such games are congestion games that have the general covering problem as underlying structure. 
In the first part of the paper, we focus on the design of utility sharing functions that maximize the price of anarchy. In particular, we construct a utility sharing function which achieves a price of anarchy of $1-\frac{1}{e}$ (Theorem 4) and show that no utility sharing function performs better (Theorem 1). To show this, we first prove a corresponding result on the price of anarchy, that depends on the maximum cardinality $k$ of the elements (Theorem 3). Surprisingly, we get matching bounds for each fixed $k$. All our results on the price of anarchy hold for pure and mixed Nash equilibria.

In the second part of the paper, we show how to use our results on the price of anarchy to construct a local search approximation algorithm for the general covering problem (Theorem 6), which runs in polynomial time, if the weights of the elements are polynomially bounded. Our hardness result in Theorem 7 shows that this restriction on the weights is necessary. For the general case, we also present a (centralized) approximation algorithm, which is based on LP-rounding and generalizes an algorithm for MAXSAT [13].

Related Work. Congestion games and variants thereof have long been used to model non-cooperative resource sharing among selfish players. Rosenthal [22] showed that congestion games always possess pure Nash equilibria. However, computing such a pure Nash equilibrium is $\mathcal{P} \mathcal{L S}$-complete [9]. The price of anarchy in congestion games has been studied extensively (see e.g. $[1,6,11,23]$ ).

The general covering problem is a natural generalization of the well-studied max- $n$-cover problem. For the max-n-cover problem, the greedy approach yields a $\left(1-\frac{1}{\mathrm{e}}\right)$-approximation [15] and no polynomial time algorithm can do better, unless $N P \subseteq T I M E\left(n^{O(\log \log n)}\right)[10]$. Applying the greedy approach to our more general problem guarantees only a $\frac{1}{2}$-approximation. For an overview on approximation algorithms for covering problems, we refer to [14, Chapter 3].

The MAXSAT problem is a special case of the generalized covering problem, where each of the $n$ collections consists of at most 2 subsets (corresponding to true/false). The power of local search for approximating MAXSAT has been studied in $[2,18]$. MAXSAT has also been considered in a game-theoretic setting as a SAT-game $[4,12]$, which is itself a special case of our covering games. Bilò [4] mainly focuses on the expressiveness of SAT-games. Mavronicolas et al. [20] concentrate on structural properties and complexity questions for a generalization of SAT-games, called weighted boolean formula games. Here, each player controls a set of variables and aims to maximize the total weight of his satisfied formulas. Giannakos et al. [12] study the price of anarchy (for pure Nash equilibria) of SAT-games under different utility sharing functions and point out the relation to approximation algorithms. Our work generalizes their results in two perspectives. First, we consider a far more general class of games, and second, we allow for mixed Nash equilibria. Moreover, in Example 1 we show that their main result [12, Thm. 5] is incorrect.

Under certain conditions on the utility sharing functions, our games fall in the class of valid utility games [26]. For such games, Vetta [26] shows that each Nash equilibrium is a $\frac{1}{2}$-approximation. Our result improves this ratio to $1-\frac{1}{\mathrm{e}}$. 
Coordination mechanisms have been introduced in [7] as a notion to improve the price of anarchy. The idea is to define local policies such that the corresponding price of anarchy is as small as possible. A few other papers follow this approach, e.g. $[3,5,16]$. Our task of designing utility sharing functions can be seen as such a coordination mechanism. However, we take the idea of coordination mechanisms one step further. In the design of our utility sharing function, the goal is not only to optimize the price of anarchy, but also to ensure that the system converges to a Nash equilibrium in polynomial time. Azar et al. [3] pursue a similar approach for the unrelated scheduling problem. However, the price of anarchy of their best coordination mechanism increases significantly by requiring a polynomial convergence.

Roadmap. The rest of the paper is organized as follows. In Section 2, we introduce covering games. Section 3 comprises our results on the price of anarchy, while Section 4 presents our approximation algorithms. We conclude in Section 5. Due to lack of space, some proofs are omitted.

\section{$2 \quad$ Model}

For any two integers $l \leq m$, denote $[m]=\{1, \ldots, m\}$ and $[l, m]=\{l, \ldots, m\}$. For a vector $\mathrm{v}=\left(v_{1}, \ldots, v_{n}\right)$, let $\mathrm{v}_{-i}=\left(v_{1}, \ldots v_{i-1}, v_{i+1}, \ldots v_{n}\right)$ and $\left(\mathrm{v}_{-i}, v_{i}^{\prime}\right)=$ $\left(v_{1}, \ldots v_{i-1}, v_{i}^{\prime}, v_{i+1}, \ldots v_{n}\right)$.

The general covering problem. In the general covering problem we are given a finite set of elements $E$ and a weight function $w: E \mapsto \mathbb{N}$ that assigns a positive integer weight $w_{e}$ to each element $e \in E$. Moreover, we are given $n$ collections $S_{1}, \ldots, S_{n}$ of subsets of $E$ where for each $i \in[n]$, the collection $S_{i} \subset 2^{E}$ is a subset of the power-set of the elements. Given such an instance our task is to choose one subset $s_{i}$ from each collection $S_{i}$ such that their union $\cup_{i \in[n]} s_{i}$ has maximum total weight, i.e. $\sum_{e \in \cup_{i \in[n]} s_{i}} w_{e}$ is maximized.

Covering Games. Each covering game has a general covering problem as an underlying structure. Here, each of the $n$ collections of subsets is controlled by a rational player, that is player $i \in[n]$ has $S_{i}$ as her strategy set. Denote $S=S_{1} \times \ldots \times S_{n}$. As for the general covering problem, each element $e \in E$ has a weight $w_{e} \in \mathbb{N}$. For any subset of the elements $E^{\prime} \subseteq E$ denote $W\left(E^{\prime}\right)=$ $\sum_{e \in E^{\prime}} w_{e}$. Let $W=W(E)$. For each element $e \in E$ denote by $k_{e}=\mid\{i \in[n]$ : $e \in s_{i}$ for some $\left.s_{i} \in S_{i}\right\} \mid$ the cardinality of $e$ which is the number of players that can possibly cover $e$. Let $k=\max _{e \in E} k_{e}$ and $k_{\min }=\min _{e \in E} k_{e}$. A covering game is a SAT-game if $\left|S_{i}\right| \leq 2$ for all player $i \in[n]$. In this case, elements correspond to clauses and players correspond to variables which can be set to true or false.

Strategies and Strategy Profiles. A pure strategy for player $i$ is some specific strategy $s_{i} \in S_{i}$, while a mixed strategy $P_{i}=\left(p\left(i, s_{i}\right)\right)_{s_{i} \in S_{i}}$ is a a probability distribution over $S_{i}$, where $p\left(i, s_{i}\right)$ denotes the probability that player $i$ chooses the pure strategy $s_{i}$.

A pure strategy profile is an $n$-tuple $\mathbf{s}=\left(s_{1}, \ldots, s_{n}\right)$ whereas a mixed strategy profile $\mathbf{P}=\left(P_{1}, \ldots, P_{n}\right)$ is represented by an $n$-tuple of mixed strategies. For a 
mixed strategy profile $\mathbf{P}$, denote by $p(\mathrm{~s})=\prod_{i \in[n]} p\left(i, s_{i}\right)$ the probability that the players choose the pure strategy profile $\mathrm{s}$.

Utility Sharing Functions. For each element $e \in E$ there is a payoff function $f_{e}$ that describes how much a player receives for covering $e$. In this paper we consider payoff functions that come from a common utility sharing function $f$. We want this function to depend only on local parameters. We consider two different kinds of utility sharing functions:

- A cardinality dependent utility sharing function depends on the number of players covering an element and the cardinality of the element, i.e. $f:[k] \times$ $[k] \mapsto \mathbb{N}$ and for all elements $e \in E$ and $j \in[k], f_{e}(j)=f\left(j, k_{e}\right) \cdot w_{e}$.

- A symmetric utility sharing function depends only on the number of players covering an element, i.e. $f:[k] \mapsto \mathbb{N}$ and for all elements $e \in E$ and $j \in[k]$, $f_{e}(j)=f(j) \cdot w_{e}$.

For both cases we assume that $f$ is non-increasing in the number of players. Moreover, we assume that $f$ does not overpay the players, i.e. $j \cdot f(j) \leq 1$ for all $j \in[k]$ in the symmetric case (and $j \cdot f(j, l) \leq 1$ for all $j \in[l], l \in[k]$ in the cardinality dependent case).

Load and Player Utilities. For a pure strategy profile s, let $\delta_{e}(\mathbf{s})=\mid\{i \in[n]$ : $\left.e \in s_{i}\right\} \mid$ denote the load on element $e \in E$, i.e. the number of players covering $e$.

Fix a pure strategy profile s. The utility $u_{i}(\mathrm{~s})$ of player $i \in[n]$ is defined by the payoff from the elements she covers. Thus, $u_{i}(\mathbf{s})=\sum_{e \in s_{i}} f_{e}\left(\delta_{e}(\mathbf{s})\right)$. For a mixed strategy profile $\mathbf{P}$, the utility of player $i \in[n]$ is $u_{i}(\mathbf{P})=\sum_{\mathbf{s} \in S} p(\mathbf{s}) \cdot u_{i}(\mathrm{~s})$.

Social Utility. Fix a pure strategy profile s. Denote by $E_{\mathrm{s}}$ the subset of elements that are covered by at least one player in s, i.e. $E_{\mathbf{s}}=\left\{e \in E: \delta_{e}(\mathrm{~s})>0\right\}$. The social utility in $\mathrm{s}$ is the total weight $W\left(E_{\mathrm{s}}\right)$ of the covered elements. We abuse notation and denote this value also as $W(\mathrm{~s})$. For a mixed strategy profile $\mathbf{P}$ the social utility $W(\mathbf{P})=\sum_{\mathbf{s} \in S} p(\mathbf{s}) \cdot W\left(E_{\mathbf{s}}\right)$ is the expected total weight of the covered elements. Throughout denote by $s^{*}$ a pure strategy profile that maximizes the total weight of the covered elements, thus, $\mathrm{s}^{*}=\arg \max _{\mathbf{s} \in S} W\left(E_{\mathbf{s}}\right)$.

Nash Equilibria and Potential Function. A mixed strategy profile $\mathbf{P}$ is a Nash equilibrium if and only if no player can increase her utility by unilaterally changing her strategy, that is, $u_{i}(\mathbf{P}) \geq u_{i}\left(\mathbf{P}_{-i}, s_{i}\right)$ for all $i \in[n]$ and $s_{i} \in S_{i}$. Depending on the type of strategy profile we distinguish between pure and mixed Nash equilibria. Given a pure strategy profile $\mathbf{s}$, a selfish step of player $i \in[n]$ is a deviation to a strategy profile $\left(\mathrm{s}_{-i}, s_{i}^{\prime}\right)$ where $u_{i}\left(\mathrm{~s}_{-i}, s_{i}^{\prime}\right)>u_{i}(\mathrm{~s})$, that is player $i$ increases her utility.

For covering games, Rosenthal's [22] exact potential function $\Phi$ implies the existence of a pure Nash equilibrium. For every pure strategy profile $\mathbf{s}$, the potential $\Phi(\mathrm{s})$ is defined by $\Phi(\mathrm{s})=\sum_{e \in E} \sum_{i=1}^{\delta_{e}(\mathrm{~s})} f_{e}(i)$. If a player performs a selfish step and increases her utility by $\Delta$, then $\Phi(\mathrm{s})$ also increases by $\Delta$.

Price of Anarchy. Let $\mathcal{G}(k)$ be the class of covering games where $k_{e} \leq k$ for all $e \in E$. Fix a utility sharing function $f$. The Price of Anarchy for $f$, denoted 
by $\mathrm{PoA}_{f}$, is the infinium, over all instances $\Gamma \in \mathcal{G}(k)$ and Nash equilibria $\mathbf{P}$, of

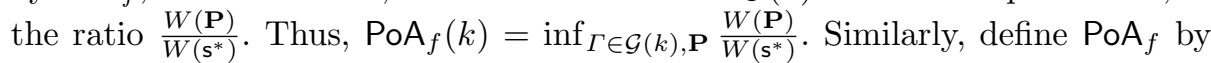
dropping the restriction on $k$.

\section{Price of Anarchy Results}

In this section, we study the price of anarchy for different utility sharing functions. We start with an upper bound that holds for all utility sharing functions.

Theorem 1. Consider the class of covering games $\mathcal{G}(k)$ with unweighted elements. Then, $\operatorname{PoA}_{f}(k) \leq 1-\frac{1}{\frac{1}{(k-1)(k-1) !}+\sum_{j \in[0, k-1]} \frac{1}{j !}}$.

This holds (a) for every cardinality dependent utility sharing function $f$, (b) even for SAT-games, if we restrict ourselves to symmetric utility sharing functions $f$.

Proof. We start by proving part (b). Given the maximum cardinality $k \in \mathbb{N}$, define a SAT-game as follows. For simplicity of description, we borrow the representation from [8] which makes use of an game graph $G$ : Elements correspond to nodes and players correspond to arcs. We allow arcs to be self loops indicating that the corresponding player has only one strategy. Every other player has exactly two strategies, namely choosing one or the other of her adjacent nodes.

The game graph is a tree which is constructed as follows: The tree consists of $k+1$ levels. We let level 0 denote the root level. At the root we have one self-loop and the root has $k-1$ children. Furthermore, every (non-root) node at level $i \in[k]$ has $k-i$ children. Figure 1 depicts our construction for $k=4$.

Denote by $E^{j}$ the set of elements at level $j \in[0, k]$. By construction, $\left|E^{0}\right|=1$ and for each $j \in[k],\left|E^{j}\right|=(k-1) \cdot \frac{(k-1) !}{(k-j) !}$.

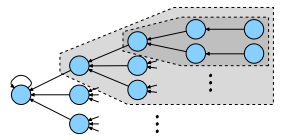

Fig. 1. The game graph for $k=4$

Let s denote the strategy profile in $\Gamma(k)$ where each player chooses the element which is closer to the root. Similarly, let $s^{*}$ be the profile where players use the elements further away from the root. It is easy to see that $\mathrm{s}$ is a pure Nash equilibrium for any utility sharing function that only depends on the number of players covering the element. In particular, for any such function, each player would receive the same payoff by switching to her alternative strategy. Moreover, for each level $j \in[0, k]$ and element $e \in E^{j}$, the profile s assigns exactly $\delta_{e}(\mathrm{~s})=k-j$ players to element $e$. So,

$$
W\left(E_{\mathrm{s}}\right)=\sum_{j \in[0, k-1]}\left|E^{j}\right|=1+\sum_{j \in[k-1]}(k-1) \cdot \frac{(k-1) !}{(k-j) !}
$$


On the other hand, in $\mathrm{s}^{*}$ there is exactly one player assigned to each element. So,

$$
W\left(E_{\mathbf{s}^{*}}\right)=\sum_{j \in[0, k]}\left|E^{j}\right|=1+\sum_{j \in[k]}(k-1) \cdot \frac{(k-1) !}{(k-j) !}
$$

It follows that

$$
\begin{aligned}
\operatorname{PoA}_{f}(k) & \leq \frac{W\left(E_{\mathrm{s}}\right)}{W\left(E_{\mathrm{s}^{*}}\right)} \\
& =\frac{1+\sum_{j \in[k-1]}(k-1) \cdot \frac{(k-1) !}{(k-j) !}}{1+\sum_{j \in[k]}(k-1) \cdot \frac{(k-1) !}{(k-j) !}} \\
& =1-\frac{1}{\frac{1}{(k-1)(k-1) !}+\sum_{j \in[0, k-1]} \frac{1}{j !}} .
\end{aligned}
$$

This finishes the proof of part (b).

For part (a) we modify our instance such that $k_{e}=k$ for all $e \in E$. For such instances there is no difference between symmetric and cardinality dependent utility sharing functions. We will add additional (single element) strategies to the strategy sets of the players. This is done in a way such that $s$ is still a Nash equilibrium and $\mathrm{s}^{*}$ still covers all elements.

For all levels $j \in[2, k]$, consider an arbitrary circular ordering of the elements in $E^{j}$. For each player $i \in[n]$, denote by $e_{i}$ the element that $i$ covers in $\mathrm{s}^{*}$. If $e_{i} \in E^{j}$ for some $j \in[2, k]$ then add the next $j-1$ elements - that follow $e_{i}$ in the ordering of $E^{j}$ - as single element strategies to $S_{i}$. Observe that $\left|S_{i}\right|=j+1$ afterwards. If we do this for all players $i \in[n]$ then we end up with an instance where $\mathrm{s}$ is still a Nash equilibrium and $k_{e}=k$ for all $e \in E$. This finishes the proof of part (a).

For SAT-games Giannakos et al. [12, Thm. 5] claim that using the cardinality dependent utility sharing function defined by $f(1, l)=1$ and $f(j, l)=\frac{1}{2(l-1)}$ for $j \geq 2$ achieves a price of anarchy of $\frac{2}{3}$. The following example shows that this does not hold:

Example 1. Given the maximum cardinality $k$, define a SAT-game with $k$ players and $k+1$ elements. We have $w_{e}=1$ for all $e \in[k-1]$ and $w_{k}=w_{k+1}=2(k-1)$. Each player $i \in[k-1]$ can either cover element $i$ or element $k$, while player $k$ can choose between the elements $k$ and $k+1$.

Let $\mathrm{s}$ be the strategy profile where each players $i \in[k]$ covers element $i$. It's not hard to see that $\mathrm{s}$ is a Nash equilibrium with $W\left(E_{\mathrm{s}}\right)=3(k-1)$. On the other hand there is a strategy profile $s^{*}$ (where only element 1 is not covered) with $W\left(\mathrm{~s}^{*}\right)=5(k-1)-1$. For $k \geq 4$, this stands in conflict to the claim in [12, Thm. $5]$; and for $k \rightarrow \infty$, we might only cover $\frac{3}{5}$ of the optimum total weight. In fact, we believe that our upper bound in Theorem 1 holds also for SAT-games with cardinality dependent utility sharing functions. 
We proceed by introducing a parameter $\chi_{f}$ of the utility sharing function $f$. This parameter is a measure on how fast the utility sharing function decreases. We will use $\chi_{f}$ in Theorem 2 to prove a general lower bound on the price of anarchy that depends on $\chi_{f}$.

Definition 1. Given a cardinality dependent utility sharing function $f$, define $\chi_{f}$ as the minimum value such that for all cardinalities $l \in[k]$ we have

$$
j \cdot f(j, l)-f(\min \{j+1, l\}, l) \leq \chi_{f} \cdot f(1, l) \quad \text { for all } j \in[l] .
$$

Theorem 2. Consider the class of covering games $\mathcal{G}(k)$. Let $f$ be a cardinality dependent utility sharing function, where $\alpha_{\min }=\min _{l \in[k]} f(1, l)$ and $\alpha_{\max }=$ $\max _{l \in[k]} f(1, l)$. Then, $\operatorname{PoA}_{\mathrm{f}}(k) \geq \frac{1}{\chi_{f}+1} \cdot \frac{\alpha_{\min }}{\alpha_{\max }}$.

Proof. Let $\mathbf{P}$ be an arbitrary mixed Nash equilibrium and $\mathbf{s}^{*}$ be an optimum pure strategy profile. Since $\mathbf{P}$ is a Nash equilibrium, it follows that $u_{i}(\mathbf{P})-$ $u_{i}\left(\mathbf{P}_{-i}, s_{i}^{*}\right) \geq 0$ for all player $i \in[n]$. So,

$$
0 \leq \sum_{i \in[n]} u_{i}(\mathbf{P})-\sum_{i \in[n]} u_{i}\left(\mathbf{P}_{-i}, s_{i}^{*}\right)=\sum_{\mathbf{s} \in S} p(\mathbf{s})\left(\sum_{i \in[n]} u_{i}(\mathbf{s})-\sum_{i \in[n]} u_{i}\left(\mathbf{s}_{-i}, s_{i}^{*}\right)\right)
$$

By definition of player utility, for any pure strategy profile s,

$$
\sum_{i \in[n]} u_{i}(\mathrm{~s})=\sum_{i \in[n]} \sum_{e \in s_{i}} f_{e}\left(\delta_{e}(\mathrm{~s})\right)=\sum_{e \in E} \delta_{e}(\mathrm{~s}) \cdot f_{e}\left(\delta_{e}(\mathrm{~s})\right)=\sum_{j \in[k]} \sum_{\substack{e \in E_{\mathrm{s}}, j \\ \delta_{e}(\mathrm{~s})=j}} j \cdot f_{e}(j) .
$$

Moreover,

$$
\begin{array}{r}
\sum_{i \in[n]} u_{i}\left(\mathbf{s}_{-i}, s_{i}^{*}\right)=\sum_{i \in[n]} \sum_{e \in s_{i}^{*}} f_{e}\left(\delta_{e}\left(\mathbf{s}_{-i}, s_{i}^{*}\right)\right) \geq \sum_{i \in[n]} \sum_{e \in s_{i}^{*}} f_{e}\left(\min \left\{k_{e}, \delta_{e}(\mathrm{~s})+1\right\}\right) \\
\geq \sum_{e \in E_{\mathrm{s}^{*}}} f_{e}\left(\min \left\{k_{e}, \delta_{e}(\mathrm{~s})+1\right\}\right)=\sum_{j=0}^{k} \sum_{\substack{e \in E_{\mathrm{s}^{*},} \\
\delta_{e}(\mathrm{~s})=j}} f_{e}\left(\min \left\{k_{e}, j+1\right\}\right)
\end{array}
$$

where the first inequality follows since $f_{e}$ is a non-increasing function and the second inequality follows since $\delta_{e}\left(\mathrm{~s}^{*}\right) \geq 1$ for all $e \in E_{\mathrm{s}^{*}}$.

So, for any pure strategy profile s, (2) and (3) imply:

$$
\begin{aligned}
& \sum_{i \in[n]} u_{i}(\mathrm{~s})-\sum_{i \in[n]} u_{i}\left(\mathbf{s}_{-i}, s_{i}^{*}\right) \\
& \leq \sum_{j \in[k]} \sum_{\substack{e \in E_{\mathbf{s}}, \delta_{e}(\mathbf{s})=j}} j \cdot f_{e}(j)-\sum_{j=0}^{k} \sum_{\substack{e \in E_{\mathbf{s}^{*},}, \delta_{e}(\mathbf{s})=j}} f_{e}\left(\min \left\{k_{e}, j+1\right\}\right) \\
& =\sum_{j \in[k]} \sum_{\substack{e \in E_{\mathrm{s}}, j \\
\delta_{e}(\mathbf{s})=j}} j \cdot f_{e}(j)-\sum_{j \in[k]} \sum_{\substack{e \in E_{\mathbf{s}^{*},}, \delta_{e}(\mathbf{s})=j}} f_{e}\left(\min \left\{k_{e}, j+1\right\}\right)-\sum_{e \in E_{\mathbf{s}^{*}} \backslash E_{\mathbf{s}}} f_{e}(1)
\end{aligned}
$$


$=\sum_{j \in[k]} \sum_{\substack{e \in E_{\mathbf{s}} \backslash E_{\mathbf{s}^{*}}, \delta_{e}(\mathbf{s})=j}} j f_{e}(j)+\sum_{j \in[k]} \sum_{\substack{e \in E_{\mathrm{s}^{*}}, \delta_{e}(\mathbf{s})=j}}\left[j f_{e}(j)-f_{e}\left(\min \left\{k_{e}, j+1\right\}\right)\right]-\sum_{e \in E_{\mathrm{s}^{*} \backslash E_{\mathrm{s}}}} f_{e}(1)$

By Definition 1 and the fact that $f_{e}$ is non-increasing, we have $j \cdot f_{e}(j) \leq$ $\left(\chi_{f}+1\right) f_{e}(1)$ for all $e \in E$ and $j \in\left[k_{e}\right]$. Using this and Definition 1, we get:

$$
\begin{aligned}
& \sum_{i \in[n]} u_{i}(\mathrm{~s})-\sum_{i \in[n]} u_{i}\left(\mathbf{s}_{-i}, s_{i}^{*}\right) \\
& \leq \sum_{j \in[k]} \sum_{\substack{e \in E_{\mathrm{s}} \backslash E_{\mathrm{s}^{*}}, \delta_{e}(\mathbf{s})=j}}\left(\chi_{f}+1\right) \cdot f_{e}(1)+\sum_{j \in[k]} \sum_{\substack{e \in E_{\mathrm{s}^{*}}, \delta_{e}(\mathrm{~s})=j}} \chi_{f} \cdot f_{e}(1)-\sum_{e \in E_{\mathrm{s}^{*}} \backslash E_{\mathrm{s}}} f_{e}(1) \\
& =\sum_{e \in E_{\mathbf{s}} \backslash E_{\mathbf{s}^{*}}}\left(\chi_{f}+1\right) \cdot f_{e}(1)+\sum_{e \in E_{\mathbf{s}^{*}} \cap E_{\mathbf{s}}} \chi_{f} \cdot f_{e}(1)-\sum_{e \in E_{\mathbf{s}^{*}} \backslash E_{\mathrm{s}}} f_{e}(1) \\
& =\sum_{e \in E_{\mathrm{s}} \backslash E_{\mathrm{s}^{*}}}\left(\chi_{f}+1\right) \cdot f_{e}(1)+\sum_{e \in E_{\mathrm{s}^{*}} \cap E_{\mathrm{s}}}\left(\chi_{f}+1\right) \cdot f_{e}(1)-\sum_{e \in E_{\mathrm{s}^{*}}} f_{e}(1) \\
& \leq\left(\chi_{f}+1\right) \cdot \alpha_{\max } \cdot W\left(E_{\mathrm{s}}\right)-\alpha_{\min } \cdot W\left(E_{\mathbf{s}^{*}}\right) .
\end{aligned}
$$

With (1) we get $0 \leq \sum_{\mathrm{s} \in S} p(\mathrm{~s})\left(\left(\chi_{f}+1\right) \cdot \alpha_{\max } \cdot W\left(E_{\mathrm{s}}\right)-\alpha_{\min } \cdot W\left(E_{\mathrm{s}^{*}}\right)\right)=$ $\left(\chi_{f}+1\right) \cdot \alpha_{\max } \cdot \sum_{\mathrm{s} \in S} p(\mathrm{~s}) \cdot W\left(E_{\mathrm{s}}\right)-\alpha_{\min } \cdot W\left(E_{\mathbf{s}^{*}}\right)$. Rearranging terms yields $\frac{W(\mathbf{P})}{W\left(\mathbf{s}^{*}\right)}=\frac{\sum_{\mathrm{s} \in S} p(\mathbf{s}) \cdot W\left(E_{\mathrm{s}}\right)}{W\left(E_{\mathrm{s}}\right)} \geq \frac{\alpha_{\min }}{\left(\chi_{f}+1\right) \cdot \alpha_{\max }}$. The theorem follows since $\mathbf{P}$ is an arbitrary Nash equilibrium.

In the following, we construct a utility sharing function such that the corresponding lower bound in Theorem 2 is maximized. Observe, that $\chi_{f}$ is independent of the values for $\alpha_{\min }$ and $\alpha_{\max }$. So, without loss of generality, we can restrict our attention to a symmetric utility sharing function $f$, where $\alpha_{\min }=\alpha_{\max }$. Our task is to construct a symmetric utility sharing function that solves the following optimization problem:

$$
\begin{array}{rr}
\operatorname{minimize} & \chi \\
\text { subject to } & j \cdot f(j)-f(j+1) \\
& \leq \chi \cdot f(1) \quad \forall j \in[k-1] \\
(k-1) f(k) & \leq \chi \cdot f(1)
\end{array}
$$

Replacing " $\leq$ " with "=" yields a homogeneous system of linear equations. The values for $\chi$ and the utility sharing function $f$ in the following theorem correspond to the solution of this system where $f(1)=1$.

Theorem 3. Given $k$ we can construct a symmetric utility sharing function $f$, such that $\operatorname{PoA}_{f}(k) \geq 1-\frac{1}{(k-1)(k-1) !}+\sum_{i=0}^{k-1} \frac{1}{i !}$.

Proof. Given $k$, let $f$ be the symmetric utility sharing function defined by

$$
f(j)=(j-1) ! \frac{\frac{1}{(k-1)(k-1) !}+\sum_{i=j}^{k-1} \frac{1}{i !}}{\frac{1}{(k-1)(k-1) !}+\sum_{i=1}^{k-1} \frac{1}{i !}} \quad \text { for all } j \in[k] .
$$


It is not hard to check that $f$ is a valid utility sharing function, i.e. $f$ is nonincreasing and $j \cdot f(j) \leq 1$ for all $j$. Moreover, $f$ satisfies the constraints in (4) for $\chi=\frac{1}{\frac{1}{(k-1)(k-1) !}+\sum_{i=1}^{k-1} \frac{1}{i !}}$. Recall that $\alpha_{\min }=\alpha_{\max }$ for symmetric utility sharing functions. The claim follows by applying Theorem 2 .

In order to construct the utility sharing function $f$ in Theorem 3 we need to know the maximum cardinality $k$ over all elements. However, since the value for $\chi$ from the proof of Theorem 3 is increasing with $k$, we can get the same lower bound if each element $e \in E$ is only aware of her own cardinality $k_{e}$. For this case, the cardinality dependent utility sharing function is defined by replacing $k$ with $k_{e}$ in (5). This implies:

Corollary 1. There exists a cardinality dependent utility sharing function $f$, such that $\mathrm{PoA}_{f}(k) \geq 1-\frac{1}{(k-1)(k-1) !}+\sum_{i=0}^{k-1} \frac{1}{i !}$.

Observe that the lower bounds on the price of anarchy in Theorem 3 and Corollary 1 match exactly the upper bound in Theorem 1.

There might also be cases where we want to use a utility sharing function that works for all $k$. For example, neither $k$ is known a priori nor the elements can determine their own cardinality. For such cases, we get:

Theorem 4. There exists a symmetric utility sharing function $f$ with $\operatorname{PoA}_{f}(k) \geq$ $1-\frac{1}{\mathrm{e}}$, which works for arbitrary $k$.

Proof. This follows by applying Theorem 3 for $k \rightarrow \infty$. In this case, $f$ reduces to $f(j)=\frac{(j-1) !}{\mathrm{e}-1}\left[\mathrm{e}-\sum_{i=0}^{j-1} \frac{1}{i !}\right]$ for all $j \in \mathbb{N}$, and $\chi=\frac{1}{\mathrm{e}-1}$.

We close this section with an alternative lower bound on the price of anarchy that depends on the maximum dimension $d_{\max }=\max _{i \in[n]}\left|S_{i}\right|$ over all players and the minimum cardinality of an element $k_{\min }$. For certain cases (e.g. if $k_{\min }>$ $\left.d_{\max }\right)$ this is better than the bound in Theorem 4 .

Theorem 5. Consider the class of covering games $\mathcal{G}$ where $k_{e} \geq k_{\min }$ for all elements $e \in E$ and $d_{\max }=\max _{i \in[n]}\left|S_{i}\right|$. Let $f$ be a cardinality dependent utility sharing function with $f(1, l)=1$ for all cardinalities $l \in[k]$. Then, $\operatorname{PoA}_{f}(\mathcal{G}) \geq$ $\frac{k_{\min }}{d_{\max }-1+k_{\min }}$.

Proof. Let $\mathbf{P}$ be an arbitrary (mixed) Nash equilibrium and $\mathrm{s}^{*}$ be an optimum pure strategy profile. Since $\mathbf{P}$ is a Nash equilibrium we have $u_{i}(\mathbf{P}) \geq u_{i}\left(\mathbf{P}_{-i}, s_{i}^{\prime}\right)$ for all $i \in[n]$ and all $s_{i}^{\prime} \in S_{i}$. By summing up over all players $i \in[n]$ and all strategies $s_{i}^{\prime} \in S_{i} \backslash\left\{s_{i}\right\}$, we get

$$
\begin{aligned}
0 & \leq \sum_{i \in[n]} \sum_{s_{i}^{\prime} \in S_{i} \backslash\left\{s_{i}\right\}} u_{i}(\mathbf{P})-\sum_{i \in[n]} \sum_{s_{i}^{\prime} \in S_{i} \backslash\left\{s_{i}\right\}} u_{i}\left(\mathbf{P}_{-i}, s_{i}^{\prime}\right) \\
& =\sum_{i \in[n]} \sum_{s_{i}^{\prime} \in S_{i} \backslash\left\{s_{i}\right\}} \sum_{\mathbf{s} \in S} p(\mathbf{s}) \cdot u_{i}(\mathbf{s})-\sum_{i \in[n]} \sum_{s_{i}^{\prime} \in S_{i} \backslash\left\{s_{i}\right\}} \sum_{\mathbf{s} \in S} p(\mathbf{s}) \cdot u_{i}\left(\mathbf{s}_{-i}, s_{i}^{\prime}\right) \\
& =\sum_{\mathbf{s} \in S} p(\mathbf{s}) \cdot\left(\sum_{i \in[n]} \sum_{s_{i}^{\prime} \in S_{i} \backslash\left\{s_{i}\right\}} u_{i}(\mathbf{s})-\sum_{i \in[n]} \sum_{s_{i}^{\prime} \in S_{i} \backslash\left\{s_{i}\right\}} u_{i}\left(\mathbf{s}_{-i}, s_{i}^{\prime}\right)\right)
\end{aligned}
$$


Now, for any pure strategy profile s, we have

$$
\begin{aligned}
& \sum_{i \in[n]} \sum_{s_{i}^{\prime} \in S_{i} \backslash\left\{s_{i}\right\}} u_{i}(\mathrm{~s})-\sum_{i \in[n]} \sum_{s_{i}^{\prime} \in S_{i} \backslash\left\{s_{i}\right\}} u_{i}\left(\mathbf{s}_{-i}, s_{i}^{\prime}\right) \\
& =\sum_{i \in[n]}\left(d_{i}-1\right) \sum_{e \in s_{i}} f_{e}\left(\delta_{e}(\mathrm{~s})\right)-\sum_{i \in[n]} \sum_{s_{i}^{\prime} \in S_{i} \backslash\left\{s_{i}\right\}} \sum_{e \in s_{i}^{\prime}} f_{e}\left(\delta_{e}\left(\mathbf{s}_{-i}, s_{i}^{\prime}\right)\right) \\
& \leq\left(d_{\max }-1\right) \sum_{e \in E_{\mathrm{s}}} \delta_{e}(\mathrm{~s}) \cdot f_{e}\left(\delta_{e}(\mathrm{~s})\right)-\sum_{i \in[n]} \sum_{s_{i}^{\prime} \in S_{i} \backslash\left\{s_{i}\right\}} \sum_{\substack{e \in s_{i}^{\prime}, e \in E_{\mathrm{s}^{*}} \backslash E_{\mathrm{s}}}} f_{e}\left(\delta_{e}\left(\mathrm{~s}_{-i}, s_{i}^{\prime}\right)\right) \\
& \leq\left(d_{\max }-1\right) \sum_{e \in E_{\mathrm{s}}} w_{e}-\sum_{i \in[n]} \sum_{s_{i}^{\prime} \in S_{i} \backslash\left\{s_{i}\right\}} \sum_{\substack{e \in s_{i}^{\prime}, e \in E_{\mathrm{s}^{*}} \backslash E_{\mathrm{s}}}} f_{e}(1) \\
& =\left(d_{\max }-1\right) \sum_{e \in E_{\mathrm{s}}} w_{e}-\sum_{e \in E_{\mathrm{s}} \backslash E_{\mathrm{s}}} k_{e} \cdot f_{e}(1) \\
& =\left(d_{\max }-1\right) \sum_{e \in E_{\mathrm{s}}} w_{e}-\sum_{e \in E_{\mathrm{s}^{*}} \backslash E_{\mathrm{s}}} k_{e} \cdot w_{e} \\
& \leq\left(d_{\max }-1\right) \cdot W\left(E_{\mathrm{s}}\right)-k_{\min } \cdot W\left(E_{\mathrm{s}^{*}} \backslash E_{\mathrm{s}}\right) \\
& \leq\left(d_{\max }-1+k_{\min }\right) \cdot W\left(E_{\mathrm{s}}\right)-k_{\min } \cdot W\left(E_{\mathrm{s}^{*}}\right) \text {. }
\end{aligned}
$$

By combining (6) and (7) we get

$$
\frac{W(\mathbf{P})}{W\left(\mathbf{s}^{*}\right)}=\frac{\sum_{\mathbf{s} \in S} p(\mathbf{s}) \cdot W\left(E_{\mathbf{s}}\right)}{W\left(E_{\mathbf{s}^{*}}\right)} \geq \frac{k_{\min }}{d_{\max }-1+k_{\min }} .
$$

The theorem follows since $\mathbf{P}$ is an arbitrary Nash equilibrium.

\section{Approximation Algorithm}

In the previous section, we have shown results on the price of anarchy for covering games. In this section, we want to use those results to construct a distributed, local-search approximation algorithm for the covering problem.

The idea of the algorithm is simple:

- Choose an appropriate utility sharing function,

- start with an arbitrary strategy profile, and

- let the players unilaterally perform selfish steps until a pure Nash equilibrium is reached.

The approximation ratio is the price of anarchy for the chosen utility sharing function. Rosenthal's potential function [22] can be used to bound the number of selfish step until a pure Nash equilibrium is reached. Unfortunately, the utility sharing functions in Theorem 3 and Theorem 4 do not provide a sub-exponential bound on the number of selfish steps, since the increase in the potential due to a single selfish step can be arbitrary small. 
To overcome this, we will design a new symmetric utility sharing function $f$, where for each element $e \in E$ the players receive strictly positive payoff only if at most a constant number $k^{\prime}$ of players cover this element, i.e. $f(j)=0$ for all $j>k^{\prime}$.

We will show that the right choice of $f$ yields a $\left(1-\frac{1}{\mathrm{e}}-\varepsilon\right)$-approximation algorithm, where $\varepsilon=\varepsilon\left(k^{\prime}\right)=o(1)$.

Theorem 6. For every constant $\varepsilon>0$ there exists a local-search approximation algorithm with approximation ratio $\left(1-\frac{1}{\mathrm{e}}-\varepsilon\right)$ that uses at most $\mathcal{O}\left(\frac{1}{\varepsilon} \cdot \log \log \frac{1}{\varepsilon}\right) \cdot W$ selfish steps.

Proof. Let $k^{\prime} \in \mathbb{N}$ be some positive integer (to be determined later) and construct $f$ as a solution to the following optimization problem:

$$
\begin{aligned}
\operatorname{minimize} & \chi \\
\text { subject to } & j \cdot f(j)-f(j+1) \leq \chi \cdot f(1) \quad \forall j \in\left[k^{\prime}-1\right] \\
k^{\prime} \cdot f\left(k^{\prime}\right) & \leq \chi \cdot f(1)
\end{aligned}
$$

For the solution of the corresponding homogeneous system of linear equations with $f(1)=1$ we get: $f(j)=(j-1) ! \frac{\sum_{i=j}^{k^{\prime}} \frac{1}{i !}}{\sum_{i=1}^{k^{\prime}} \frac{1}{i !}}$ for all $j \in\left[k^{\prime}\right]$ and $f(j)=0$ for $j>k^{\prime}$. It is not hard to check that $f$ is a valid utility sharing function, i.e. $f$ is non-increasing and $j \cdot f(j) \leq 1$ for all $j$.

Observe, that $f(1)=1, k^{\prime} \cdot f\left(k^{\prime}\right)=\frac{1}{\sum_{i=1}^{k^{\prime}} \frac{1}{i !}}$ and $f(j+1)=j ! \frac{\sum_{i=j}^{k^{\prime}} \frac{1}{i !}-\frac{1}{j !}}{\sum_{i=1}^{k^{\prime}} \frac{1}{i !}}=$ $j \cdot f(j)-\frac{1}{\sum_{i=1}^{k^{\prime}} \frac{1}{i !}}$. Thus, $f$ satisfies the constraints in (8) for $\chi=\frac{1}{\sum_{i=1}^{k^{\prime}} \frac{1}{i !}}$. Applying Theorem 2 yields

$$
\mathrm{PoA}_{f} \geq 1-\frac{1}{\sum_{i=0}^{k^{\prime}} \frac{1}{i !}}=1-\frac{1}{\mathrm{e}}-\varepsilon\left(k^{\prime}\right)
$$

where $\varepsilon\left(k^{\prime}\right)=\frac{1}{\sum_{i=0}^{k^{\prime}} \frac{1}{i !}}-\frac{1}{\mathrm{e}}=\frac{\sum_{i=k^{\prime}+1}^{\infty} \frac{1}{i !}}{\mathrm{e} \cdot \sum_{i=0}^{k^{\prime}} \frac{1}{i !}}=\frac{\sum_{i=k^{\prime}+1}^{\infty} \frac{k^{\prime} !}{i !}}{\mathrm{e} \cdot \sum_{i=0}^{k^{\prime}} \frac{k^{\prime} !}{i !}}=\Theta\left(\frac{1}{\left(k^{\prime}+1\right) !}\right)$. So for every constant $\varepsilon$ we choose $k^{\prime}=\Theta\left(\frac{\log \left(\frac{1}{\varepsilon}\right)}{\log \log \left(\frac{1}{\varepsilon}\right)}\right)$. We will now bound the maximum number of selfish steps. To do so, we will first show an upper bound on Rosenthal's potential function $\Phi(\mathrm{s})$. Afterwards, we show a lower bound on the increase in $\Phi$ due to a selfish step. For any pure strategy profile s we have

$$
\Phi(\mathrm{s})=\sum_{e \in E} \sum_{i=1}^{\delta_{e}(s)} f_{e}(i) \leq \sum_{e \in E} \sum_{i=1}^{k^{\prime}} f_{e}(i) \leq \sum_{e \in E} \sum_{i=1}^{k^{\prime}} \frac{1}{i} \cdot w_{e}=H\left(k^{\prime}\right) \cdot W
$$

where $H\left(k^{\prime}\right)$ is the harmonic number of order $k^{\prime}$.

Recall, that $w_{e} \in \mathbb{N}$ for all $e \in E$. Moreover, for all $j \in\left[k^{\prime}\right], f(j)$ is an integer multiple of $\frac{1}{\sum_{i=1}^{k^{\prime}} \frac{k^{\prime} !}{i !}}$. To see this multiply the enumerator and denominator by $k^{\prime}$ ! and observe that both become integer. So if a player improves, then she improves by at least $\frac{1}{\sum_{i=1}^{k^{\prime}} \frac{k^{\prime} !}{i !}}$. Using the property of Rosenthal's exact potential 
function (cf. Sec. 2), it follows that each selfish step increases $\Phi$ by at least $\frac{1}{\sum_{i=1}^{k^{\prime}} \frac{k^{\prime} !}{i !}}=\frac{1}{\left\lfloor(\mathrm{e}-1) k^{\prime} !\right]}$. So the number of selfish steps is upper bounded by $\lfloor(\mathrm{e}-$ 1) $\left.k^{\prime} !\right\rfloor \cdot H\left(k^{\prime}\right) \cdot W=\mathcal{O}\left(k^{\prime} ! \cdot \log k^{\prime}\right) \cdot W=\mathcal{O}\left(\frac{1}{\varepsilon} \cdot \log \log \frac{1}{\varepsilon}\right) \cdot W$

Theorem 6 implies a polynomial time $\left(1-\frac{1}{\mathrm{e}}-\varepsilon\right)$-approximation algorithm for the case that the total weight $W$ is polynomially bounded. This includes the important case of unweighted elements, where $w_{e}=1$ for all $e \in E$. In the following theorem we show that this restriction on $W$ is necessary, since for arbitrary weights the problem of computing a pure Nash equilibrium is $\mathcal{P} \mathcal{L} \mathcal{S}$ complete (see [17] for an introduction to the complexity class $\mathcal{P} \mathcal{L S}$ ).

Theorem 7. Consider the class of covering games with arbitrary weights. Then, for every symmetric utility sharing function with $f(1)>f(2)$, it is $\mathcal{P} \mathcal{L} \mathcal{S}$-complete to compute a pure Nash equilibrium. This holds even for SAT-games.

Proof. We reduce from MaxCut [24]: Given an undirected graph $G=(V(G), E(G))$ with non-negative edge weights. Find a partition of $V(G)$ into two subsets $L$ and $R$ such that the total weight of edges between $L$ and $R$ cannot be increased by flipping a single node.

Given an instance of MAxCUT we construct an instance of the covering game as follows: Each node $u \in V(G)$ corresponds to a player $u$. For each edge $(u, v) \in$ $E(G)$ of weight $w$ we introduce two elements $e_{(u, v)}^{L}$ and $e_{(u, v)}^{R}$ with weight $w$. Each player $u$ has strategy set $S_{u}=\left\{L_{u}, R_{u}\right\}$ where $L_{u}=\left\{e_{(u, v)}^{L}:(u, v) \in E(G)\right\}$ and $R_{u}=\left\{e_{(u, v)}^{R}:(u, v) \in E(G)\right\}$. A player $u$ choosing $L_{u}$ (resp. $R_{u}$ ) corresponds to assigning node $u$ to $L$ (resp. $R$ ). It is not hard to see that if a player improves her payoff by $\Delta$ by unilaterally changing her strategy, then the total weight of the corresponding cut increases by $\frac{\Delta}{f(1)-f(2)}$, and vice versa. The claim follows.

In the following we discuss an alternative approach for approximating the general covering problem which works for arbitrary weights. This approach is a generalization of the corresponding algorithm for MAXSAT from [13] (see [25, Chapter 16.3] for a textbook description) which is based on LP-rounding.

For the following integer program representation of the general covering problem, we introduce two sets of indicator variables. For each element $e \in E$ we have a variable $z_{e} \in\{0,1\}$ that can only be set to 1 if $e$ is covered at least once. Moreover, for each player $i$ and strategy $s_{i} \in S_{i}$, we have a variable $y_{i, s_{i}} \in\{0,1\}$ which is 1 if player $i$ chooses strategy $s_{i}$.

$$
\begin{aligned}
& \operatorname{maximize} \sum_{e \in E} w_{e} \cdot z_{e} \\
& \text { subject to } \sum_{i \in[n]} \sum_{\substack{s_{i} \in S_{i}: \\
e \in s_{i}}} y_{i, s_{i}} \geq z_{e} \quad \forall e \in E \\
& \sum_{s_{i} \in S_{i}} y_{i, s_{i}}=1 \quad \forall i \in[n] \\
& z_{e} \in\{0,1\} \quad \forall e \in E \\
& y_{i, s_{i}} \in\{0,1\} \quad \forall i \in[n], s_{i} \in S_{i}
\end{aligned}
$$


For the general case, we present a (centralized) approximation algorithm, which is based on LP-rounding and generalizes an algorithm for MaxSat [13].

Theorem 8. There exists a (centralized) polynomial-time (1- $\left.\frac{1}{\mathrm{e}}\right)$-approximation algorithm for the general covering problem.

Proof. Consider the LP-relaxation of (9) where the $z$ and $y$ variables can hold arbitrary values between 0 and 1 .

The algorithm is simple: Solve the LP-relaxation, denote $z^{*}, y^{*}$ as the optimum solution. Let player $i$ choose strategy $s_{i}$ with probability $y_{i, s_{i}}^{*}$.

Fix an element $e \in E$. For each player $i \in[n]$, denote $y_{i e}=\sum_{s_{i} \in S_{i}: e \in s_{i}} y_{i, s_{i}}$. Then the probability that $e$ is covered in $\mathrm{y}^{*}$ is

$$
\begin{aligned}
1-\prod_{i \in[n]}\left(1-y_{i e}\right) & \geq 1-\left(\frac{\sum_{i \in[n]: y_{i e}>0}\left(1-y_{i e}\right)}{k_{e}}\right)^{k_{e}} \\
& \geq 1-\left(1-\frac{z_{e}^{*}}{k_{e}}\right)^{k_{e}} \\
& \geq 1-\left(\frac{1}{\mathrm{e}}\right)^{z_{e}^{*}} \\
& \geq\left(1-\frac{1}{\mathrm{e}}\right) \cdot z_{e}^{*} .
\end{aligned}
$$

Here, the first inequality uses the arithmetic-geometric mean inequality, the second inequality uses the constraint on $z_{e}$ from the LP, the third inequality is a standard property of e, and the last inequality follows by concavity.

It follows that the expected total weight of the covered elements is lower bounded by

$$
\sum_{e \in E}\left(1-\frac{1}{\mathrm{e}}\right) \cdot z_{e}^{*} \cdot w_{e}=\left(1-\frac{1}{\mathrm{e}}\right) \cdot \mathrm{OPT}^{*}
$$

where OPT* is the value of the solution to the LP-relaxation, which is at least the value of an optimum integral solution. The algorithm can be derandomized via method of conditional expectation [25, Chapter 16.2]. The claim follows.

\section{Conclusion}

In this paper we use game theoretic concepts for the design of new local search approximation algorithms for a very general covering problem. Our approach is to design player payoff functions that minimize the price of anarchy and guarantee that any sequence of unilateral improvements by the players is of polynomial length. For the covering problem this yields essentially the best possible approximation ratio. 
For future work, we propose to study how far such ideas can be utilized to get new local search approximation algorithms also for other interesting optimization problems. Certainly, our approach will not always yield the best possible approximation ratio. This gives rise to the new interesting concept of selfish approximation ratio, i.e. the best possible approximation ratio that can be achieved by selfish players.

\section{Acknowledgement}

We thank Adrian Vetta and Thomas Sauerwald for many helpful discussions and comments on the topic.

\section{References}

1. Aland, S., Dumrauf, D., Gairing, M., Monien, B., Schoppmann, F.: Exact Price of Anarchy for Polynomial Congestion Games. In Durand, B., Thomas, W., eds.: Proceedings of the 23rd International Symposium on Theoretical Aspects of Computer Science (STACS'06). Lecture Notes in Computer Science, Vol. 3884, Springer Verlag (2006) 218-229

2. Alimonti, P.: New Local Search Approximation Techniques for Maximum Generalized Satisfiability Problems. In: In Proceedings of CIAC'94. Lecture Notes in Computer Science, Vol. 778, Springer Verlag (1994) 40-53

3. Azar, Y., Jain, K., Mirrokni, V.S.: (Almost) optimal coordination mechanisms for unrelated machine scheduling. In: Proceedings of the 19th Annual ACM-SIAM Symposium on Discrete Algorithms (SODA'08). (2008) 323-332

4. Bilò, V.: On Satisfiability Games and the Power of Congestion Games. In: In Proceedings of 3rd International Conference on Algorithmic Aspects in Information and Management (AAIM'07). Lecture Notes in Computer Science, Vol. 4508, Springer Verlag (2007) 295-304

5. Chen, H.L., Roughgarden, T., Valiant, G.: Designing Networks with Good Equilibria. In: Proceedings of the 19th Annual ACM-SIAM Symposium on Discrete Algorithms (SODA'08). (2008) 854-863

6. Christodoulou, G., Koutsoupias, E.: The Price of Anarchy of Finite Congestion Games. In: Proceedings of the 37th Annual ACM Symposium on Theory of Computing (STOC'05). (2005) 67-73

7. Christodoulou, G., Koutsoupias, E., Nanavati, A.: Coordination Mechanisms. In Diaz, J., Karhumäki, J., Lepistö, A., Sannella, D., eds.: Proceedings of the 31st International Colloquium on Automata, Languages, and Programming (ICALP'04). Lecture Notes in Computer Science, Vol. 3142, Springer Verlag (2004) 345-357

8. Elsässer, R., Gairing, M., Lücking, T., Mavronicolas, M., Monien, B.: A Simple Graph-Theoretic Model for Selfish Restricted Scheduling. In Deng, X., Ye, Y., eds.: Proceedings of the 1st International Workshop on Internet and Network Economics (WINE'05). Lecture Notes in Computer Science, Vol. 3828, Springer Verlag (2005) 195-209

9. Fabrikant, A., Papadimitriou, C.H., Talwar, K.: The Complexity of Pure Nash Equilibria. In: Proceedings of the 36th Annual ACM Symposium on Theory of Computing (STOC'04). (2004) 604-612 
10. Feige, U.: A Threshold of $\ln n$ for Approximating Set Cover. Journal of the ACM 45 (1998) 314-318

11. Gairing, M., Schoppmann, F.: Total Latency in Singleton Congestion Games. In Deng, X., (Graham), F.C., eds.: Proceedings of the 3rd International Workshop on Internet and Network Economics (WINE'07). Lecture Notes in Computer Science, Vol. 4858, Springer Verlag (2007) 381-387

12. Giannakos, A., Gourvès, L., Monnot, J., Paschos, V.T.: On the Performance of Congestion Games for Optimum Satisfiability Problems. In Deng, X., (Graham), F.C., eds.: Proceedings of the 3rd International Workshop on Internet and Network Economics (WINE'07). Lecture Notes in Computer Science, Vol. 4858, Springer Verlag (2007) 220-231

13. Goemans, M.X., Williamson, D.P.: New $\frac{3}{4}$-approximation algorithms for the maximum satisfiability problem. SIAM Journal on Discrete Mathematics 7 (1994) 656-666

14. Hochbaum, D.S.: Approximation Algorithms for NP-Hard Problems. PWS Publishing Company (1997)

15. Hochbaum, D.S., Pathria, A.: Analysis of the Greedy Approach in Problems of Maximum $k$-coverage. Naval Research Logistics 45(6) (1998) 615-627

16. Immorlica, N., Li, L., Mirrokni, V.S., Schulz, A.: Coordination Mechanisms for Selfish Scheduling. In Deng, X., Ye, Y., eds.: Proceedings of the 1st International Workshop on Internet and Network Economics (WINE'05). Lecture Notes in Computer Science, Vol. 3828, Springer Verlag (2005) 55 - 69

17. Johnson, D.S., Papadimitriou, C.H., Yannakakis, M.: How Easy is Local Search? Journal of Computer and System Sciences 37(1) (1988) 79-100

18. Khanna, S., Motwani, R.: On Syntactic versus Computational Views of Approximability. SIAM Journal on Computing 28(1) (1998) 164-191

19. Koutsoupias, E., Papadimitriou, C.H.: Worst-Case Equilibria. In Meinel, C., Tison, S., eds.: Proceedings of the 16th International Symposium on Theoretical Aspects of Computer Science (STACS'99). Lecture Notes in Computer Science, Vol. 1563, Springer Verlag (1999) 404-413

20. Mavronicolas, M., Monien, B., Wagner, K.W.: Weighted Boolean Formula Games. In Deng, X., (Graham), F.C., eds.: Proceedings of the 3rd International Workshop on Internet and Network Economics (WINE'07). Lecture Notes in Computer Science, Vol. 4858, Springer Verlag (2007) 469 - 481

21. Nash, J.F.: Non-Cooperative Games. Annals of Mathematics 54(2) (1951) 286-295

22. Rosenthal, R.W.: A Class of Games Possessing Pure-Strategy Nash Equilibria. International Journal of Game Theory 2 (1973) 65-67

23. Roughgarden, T.: Intrinsic Robustness of the Price of Anarchy. In: Proceedings of the 41st Annual ACM Symposium on Theory of Computing (STOC'09). (2009) $513-522$

24. Schäffer, A.A., Yannakakis, M.: Simple Local Search Problems that are Hard to Solve. SIAM Journal of Computing 20(1) (1991) 56-87

25. Vazirani, V.V.: Approximation Algorithms. Springer Verlag (2003)

26. Vetta, A.: Nash Equilibria in Competitive Societies, with Applications to Facility Location, Traffic Routing and Auctions. In: Proceedings of the 43rd Annual Symposium on Foundations of Computer Science (FOCS'02). (2002) 416-425 